International Journal of Engineering \& Technology, $7(1.1)(2018) 414-417$
SPC
International Journal of Engineering \& Technology
Website www.sciencepubco.com/index.php/IJET
Research paper

\title{
An efficient method for early stage detection of diabetic retinopathy
}

\author{
S. D. Shirbahadurkar ${ }^{1 *}$, V. M. Mane ${ }^{2}$, D. V. Jadhav ${ }^{3}$ \\ ${ }^{1}$ Department of Electronics and Telecommunication Engineering, Zeal College of Engineering and Research, Pune \\ ${ }^{2}$ Research Scholar, Department of Electronics and Telecommunication Engineering, Rajarshi Shahu College of Engineering, Pune \\ ${ }^{3}$ Department of Electronics Engineering, Government Polytechnic, Ambad \\ *Corresponding author E-mail: shirsd112@yahoo.in
}

\begin{abstract}
Diabetic Retinopathy (DR) is one of the leading causes of blindness. The early detection and treatment of DR is significant to save the human vision. The presence of microaneurysms (MAs) is the first sign of the disease. The correct identification of MAs is an essential for finding of DR at the early stages. In this paper, we propose a three phase system for efficient recognition of MAs. The tentative MA lesions are recovered from the fundus image in the first stage. To accurately classify an extracted candidate region into MA or non-MA, the second stage prepares an attribute vector for each tentative MA lesion based on shape, intensity and statistical properties. The third stage is a classification step to classify as MAs and Non-MAs for early stage detection of DR. We present a holoentropy enabled decision tree classifier which combines entropy and total correlation. The best feature for decision tree is selected based on holoentropy to enhance the correctness of the classification. The implemented system is experimented using fundus image database DIARETDB1. The proposed method achieved an overall accuracy of $97.67 \%$. The proposed system has detected the MAs with higher performance using simple features and holoentropy based decision tree classifier. The proposed system is suitable for early stage detection of DR through the accurate identification of MAs.
\end{abstract}

Keywords: Fundus Image; Features; Entropy; Classification; Diabetic Retinopathy.

\section{Introduction}

Diabetic Retinopathy (DR) is one of the major sources of the sightlessness which is caused because of prolonged diabetes mellitus. It is estimated that, nearly 220 million people are affected by diabetes [1]. However, proper screenings, early detection and appropriate treatment limit the visual impairments [2]. Different abnormalities such as microaneurysms (MA), internal bleeding, hard exudates and cotton wools appear as clinical symptoms of non-proliferative DR (NPDR) as shown in Fig. 1. During the screening process, fundus images of the retina are taken by fundus camera for the purpose of detection of DR. The presence of MAs in the retinal fundus images is an early indicator for detection of DR. The automated segmentation and identification of MAs can aid in screening programs for DR diagnosis. Thus, an automated DR screening system is essential to reduce the time required by specialists for manual intervention. This will enhance the resourcefulness of the eye care delivery even at the underserved places [3]. The accurate recognition of MAs from the retinal fundus images is necessary to detect DR at early stage.

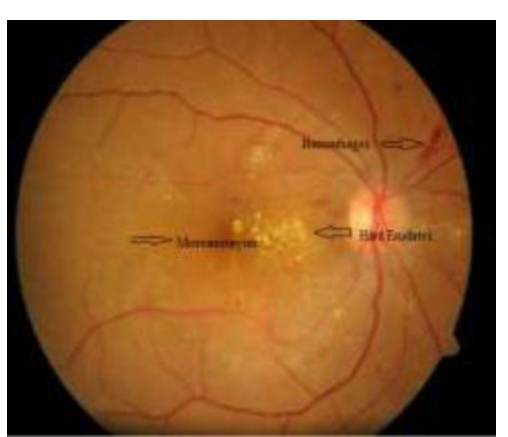

Fig. 1: NPDR Fundus Image.

The accurate detection of MAs is a difficult task because of the variations in the appearance of MAs in the retinal images [4]. A novel region-growing based on gradient values has been introduced by Fleming et al. [5]. The paraboloid parameters were used to compute the features which are used in the classification phase. Zhang et al. [6] applied multiple Gaussian filters at multiple scales. They computed the maximum response to generate a probability map of the tentative occurrence of MAs. The initial set of MA candidates was produced using thresholding of probability map. The final classification was performed using a rule-based classifier using 30 features. Sánchez et al. [7] used a mixture model-based clustering technique to detect the MA candidate regions. The technique fits three normal distribution histograms corresponding to foreground, background and outliers. The foreground histogram pixels were considered as the set of MA candidate regions. The logistic regression was used to classify each MA region. The tenta- 
tive candidate lesions were detected using simple thresholding by Giancardo et al. [8]. An initial set of 31 features were extracted. The number of the features was reduced to 10 by using principle component analysis, and this reduced version of features was used to perform the classification. Radon transform was used to extract the features of the tentative candidates and a support vector machine (SVM) classifier was utilized to perform the final classification. Sinthanayothin et al. [9] enhanced red lesions in the image using moat operator. The blood vessel regions were then removed from the segmented image to produce the final set of candidates. Ram et al. [10] used a dual classifier to classify the tentative candidates. The tentative candidate lesions were detected using a simple thresholding operation. The first classification stage was applied to segment MAs from vessels. The second classification stage was applied to further separate MAs from other types of noise. Antal and Hajdu [11] presented a DR screening method. They extracted the features using various preprocessing algorithms. They combined different classifiers for the final decision of DR. Akram et al [12] extracted the candidate lesions using Gabor filter bank. The final classification was based on hybrid classifier.

A system for efficient and accurate detection of MAs using holoentropy based decision tree classifier is presented in this paper. Total eleven optimal attributes are extracted for each candidate MA. The extracted attributes are used to build a holoentropy enabled decision tree classifier. The final classification as MAs and Non MAs is performed using predicted holoentropy enabled decision tree classifier. The proposed methodology is enlightened in Section II. Section III elaborates the experimental results. The conclusion of the paper is made in Section IV.

\section{Proposed methodology}

The presence of MAs in the fundus image is the first sign of DR. Hence MA detection is a critical step in early stage detection of DR. The automatic system for detection of MAs to reduce a load on ophthalmologists and to make it cost and time efficient is required [2]. Accordingly, a method is developed for automatic identification of MAs from retinal fundus images. The classifier, holoentropy based decision tree (HDT) is utilized for identification of MAs. The proposed system has been implemented in three stages: extraction of candidate regions, formation of attribute vector and classification. The block diagram of the presented method is shown in the Fig 2.

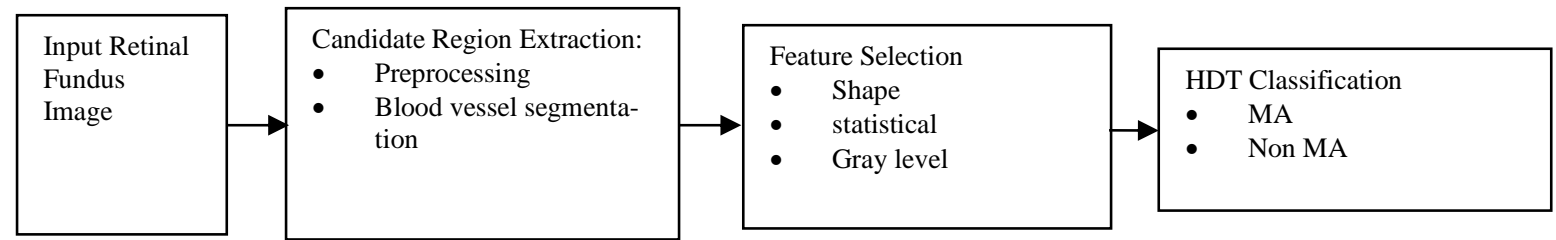

Fig. 2: Block Diagram of the Proposed System.

\subsection{Candidate region extraction}

The first step extracts the tentative MAs from the input retinal fundus image. The input color retinal image is changed to green channel. The green channel image provides the most excellent contrast. The green channel easily makes a distinction among background and foreground regions. The adaptive histogram equalization technique is used to enhance the contrast. The blood vessels are enhanced using matched filter approach [13-14]. The segmentation of blood vessels is achieved by local entropy thresholding [15]. The length filtering is applied to remove misclassified and secluded pixels. The candidate lesions are obtained by taking difference of the length filtered image and local entropy threshold image [16]. The remained isolated objects give tentative lesions for MAs. All such isolated objects are referred as candidate MA lesions from which optimal features are extracted.

\subsection{Feature selection}

The extracted candidate region contains all possible MA regions. It is necessary to differentiate between MAs and non MAs. The true MAs have noticeable distinctiveness like, dark red in color and circular in shape. From the extracted tentative candidate MA lesions, a feature vector is created. The features are extracted for each candidate MA based on statistical, geometrical and intensity features.

\subsection{Classification using HDT}

The feature vector is generated with extracted eleven features which are used for MA detection and classification. Decision tree is a simple and practical tool for classification. The decision tree develops the system for classification with minimum computational processes. It is constructed using main steps such as selection of the best feature as a node, split the feature to progress the tree and finally label the class. The node with best feature is selected using holoentropy instead of entropy. The holoentropy combines entropy and total correlation [17]. The holoentropy has been calculated for each feature from the feature vector. The feature having highest holoentropy is chosen as the best feature to construct the decision tree [18]. The holoentropy HLE $\left(\mathrm{a}_{\mathrm{i}}\right)$ is calculated as given in Eq. (1). The reverse sigmoid function of the entropy is used to weight the entropy as in Eq. (1).

$$
\begin{aligned}
& \operatorname{HLE}\left(\text { attribute }_{i}\right)= \\
& 2\left(1-\frac{1}{1+\exp \left(- \text { Entropy }_{\left(\text {attribute }_{i}\right)}\right)}\right) \cdot \text { Entropy }_{\left(\text {attribute }_{i}\right)}
\end{aligned}
$$

The splitting of the selected best feature to construct the decision tree has performed by selecting the best possible split. This process is performed by utilizing holoentropy information gain HLEIG and conditional holoentropy (CHLE) as given in Eq. (2) and (3). The process is executed to all the samples recursively [18].

$\operatorname{HLEIG}\left(a_{i}, a_{j}\right)=\operatorname{HLE}\left(a_{i}\right)-\operatorname{CHLE}\left(a_{i}, a_{j}\right)$

Where

$\operatorname{CHLE}\left(a_{i}, a_{j}\right)=\sum_{i=0}^{\mu\left(a_{i}\right)} P_{i} \cdot \operatorname{HLE}\left(a_{i}, a_{j}\right)$

The labeling of the last node that is leaf node is performed by selecting the group to which the large amount of data fulfilled with the same node. The output of each step implemented is as shown in Fig. 3. The HDT visualization of DIARETDB1 database is shown in Fig.4. 
(A)

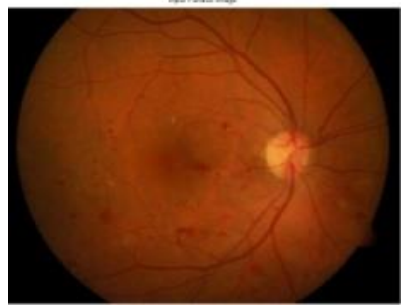

(D)

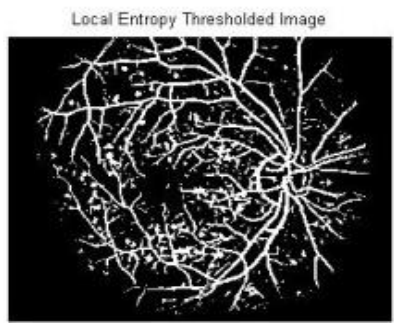

(G)

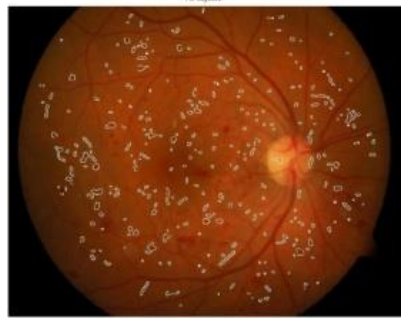

(B)

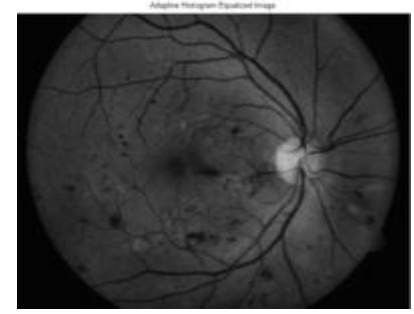

(E)

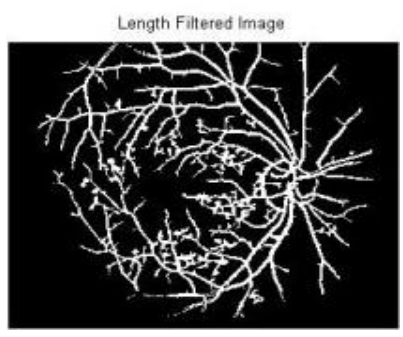

(H)
(C)

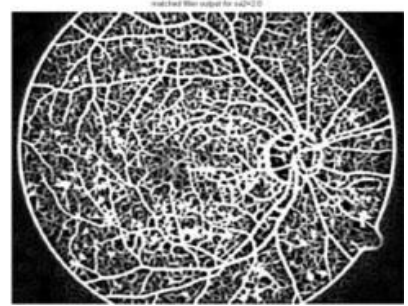

(F)

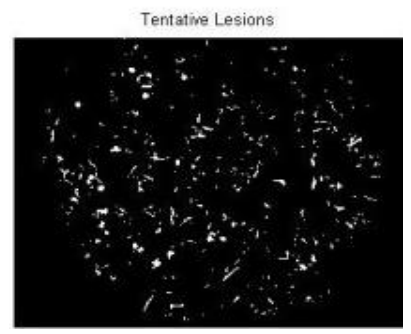

Fig. 3: A) Input Fundus Image. B) Preprocessed Green Channel Image. C) Matched Filter Output. D) Local Entropy Thresholding. E) Length Filtering. F) Extracted Candidate Lesions. G) Labeling. H) Final Mas Detected from HDT Classifier.

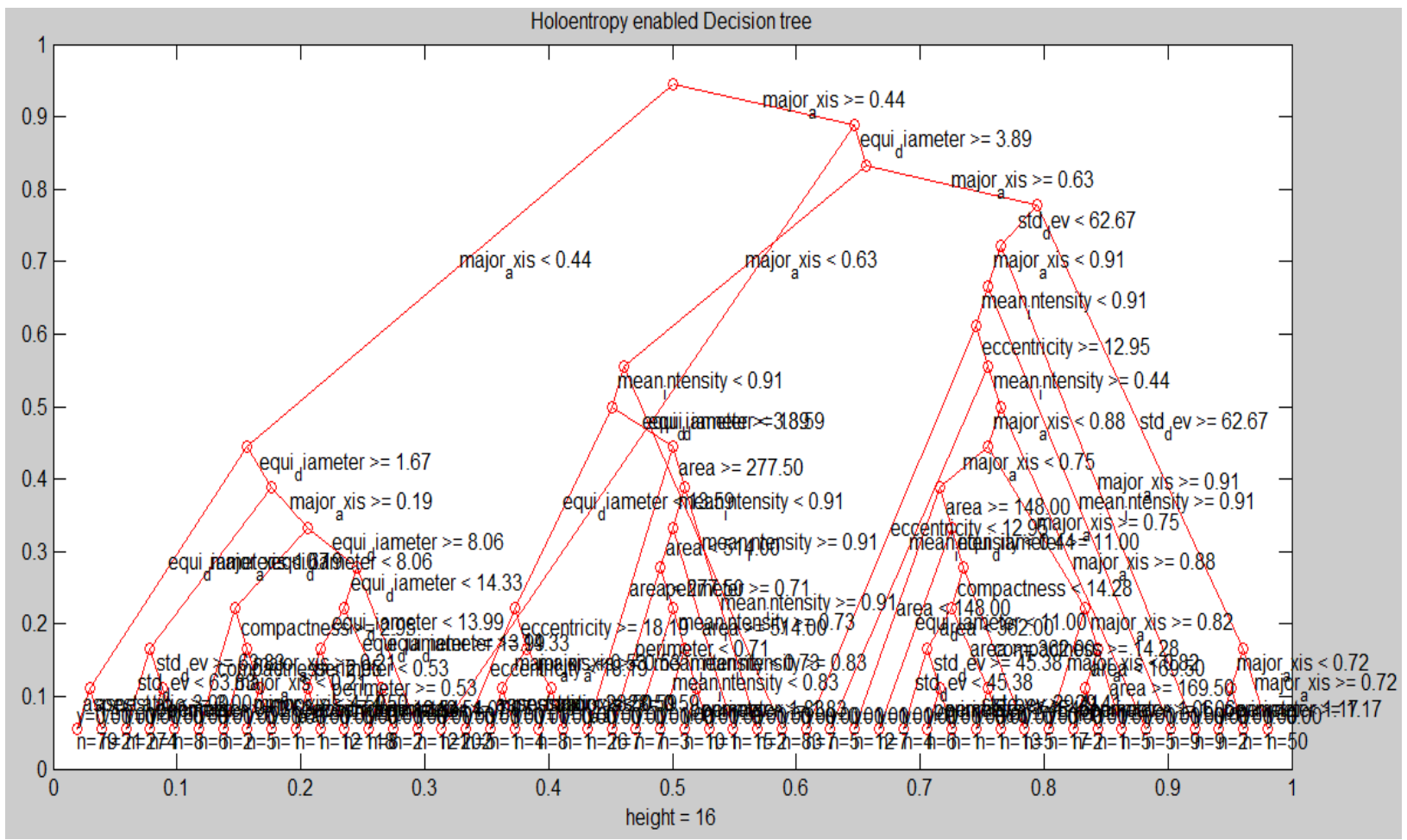

Fig. 4: Visualization of HDT.

\section{Experimental results}

The experimental evaluation and the performance of the proposed MA detection using holoentropy enabled decision tree classifier for early stage detection of DR is presented here. The proposed method has been executed using MatLab R2013b with a desktop computer configuration of 4GB RAM and 64 bit OS. The work has been experimented over a large-scale database which is publically available standard DR database DIARETDB1 [19]. The database DIARETDB1 provides 89 color retina images. Out of theses 89 images, the eighty four images have signs of the NPDR and the rest of the 5 images are normal images. The total number of lesions present in the 89 images is 1304 . The dataset is provided with its ground-truth, the locations of the abnormalities in the retinal fundus 
image. The proposed classifier is trained using 652 lesions and testing has been performed on remained 652 lesions. The dataset used for testing consists of 220 MA lesions and 432 non MA lesions. The performance has been evaluated based on the ground truth provided using performance metrics as mentioned in Table 1. The sensitivity defines the correctly classifying the MA lesions; the specificity is the number to correctly classifying non MA lesions. The true results are the accuracy. True positive TP is defined as correctly classified lesions and false negative FN denotes the incorrectly rejected lesions. The true negative - $\mathrm{TN}$, is the correctly rejected lesions. The incorrectly detected lesion is the false positiveFP.

Table 1: Performance of the Proposed System

\begin{tabular}{ll}
\hline Factors & Performance \\
\hline TP & 209 \\
TN & 428 \\
FP & 4 \\
FN & 11 \\
Sensitivity & 95 \\
Specificity & 99.07 \\
Accuracy & 97.69 \\
\hline
\end{tabular}

\section{Conclusion}

An efficient method to detect of MAs for early stage detection of DR is presented in this paper. This system has been implemented in three stages such as extraction of candidate regions, formation of feature vector and classification. The color retinal image is converted to green channel. The adaptive histogram equalization technique is used to enhance the contrast. The blood vessels are enhanced and removed using matched filter and local entropy thresholding. The length filtering is applied to remove misclassified and isolated pixels. The features are extracted for each candidate MA based on statistical, geometrical and intensity features. A holoentropy based decision tree has been developed as a classifier for automatic detection of microaneurysms. The decision systems were generated with the help of the features which is used to form feature vector. The proposed system is analyzed using sensitivity, specificity and accuracy. The publically available dataset is used for the assessment of the system. The results demonstrate that the proposed system using simple features and holoentropy based decision tree classifier has detected the MAs with higher efficiency. The proposed system is appropriate for early detection of diabetic retinopathy through the accurate identification and classification of microaneurysms.

\section{References}

[1] WHO, 2011. http://www.who.int/mediacentre/factsheets/fs312/en/

[2] Mane VM \& Jadhav DV, "Progress towards automated early stage detection of diabetic retinopathy: Image Analysis Systems and Potential", Medical and Biological Engineering, Vol.34, No.6, (2014), pp.520-527.

[3] Fleming AD, Philip S, Goatman KA, Prescott GJ, Sharp PF \& Olson JA, "The evidence for automated grading in diabetic retinopathy screening", Curr. Diabetes Rev, Vol.7, No.4, (2011), pp.246252. https://doi.org/10.2174/157339911796397802.

[4] Lazar I \& Hajdu A, "Retinal Microaneurysm Detection Through Local Rotating Cross-Section Profile Analysis”,IEEE Transactions on Medical Imaging, Vol.32, No.2, (2013), pp.400-407. https://doi.org/10.1109/TMI.2012.2228665.

[5] Fleming AD, Philip S, Goatman KA, Olson JA \& Sharp PF, “Automated microaneurysm detection using local contrast normalization and local vessel detection", IEEE Transactions on Medical Imaging, Vol.25, No.9, (2006), pp.1223-1232. https://doi.org/10.1109/TMI.2006.879953.

[6] Zhang B, Wu X, You J, Li Q \& Karray F, "Detection of microaneurysms using multi-scale correlation coefficients," Pattern Recognition, Vol.43, No.6, (2010), pp.2237-2248. https://doi.org/10.1016/j.patcog.2009.12.017.

[7] Sánchez CI, Hornero R, Mayo A \& García M, "Mixture modelbased clustering and logistic regression for automatic detection of microaneurysms in retinal images", SPIE medical imaging, Vol.7260, (2009). https://doi.org/10.1117/12.812088.

[8] Giancardo L, Meriaudeau F, Karnowski TP, Li Y, Tobin Jr KW \& Chaum E, "Microaneurysm detection with radon transform-based classification on retina images", Annual International Conference of the IEEE Engineering in Medicine and Biology Society, EMBC, (2011), https://doi.org/10.1109/IEMBS.2011.6091562.

[9] Sinthanayothin C, Boyce JF, Williamson TH, Cook HL, Mensah, E, Lal S \& Usher D, "Automated detection of diabetic retinopathy on digital fundus images", Diabetic medicine, Vol.19, No.2, (2002), pp.105-112. https://doi.org/10.1046/j.1464-5491.2002.00613.x.

[10] Ram K, Joshi GD \& Sivaswamy J, “A Successive ClutterRejection-Based Approach for Early Detection of Diabetic Retinopathy", IEEE Transactions on Biomedical Engineering, Vol.58, No.3, (2011) https://doi.org/10.1109/TBME.2010.2096223.

pp.664-673.

[11] Antal B \& Hajdu A, "An ensemble-based system for automatic screening of diabetic retinopathy", Knowledge-based systems, Vol.60, (2014), https://doi.org/10.1016/j.knosys.2013.12.023.

[12] Akram MU, Khalid S \& Khan SA, "Identification and classification of microaneurysms for early detection of diabetic retinopathy", Pattern Recognition, Vol.46, No.1, (2013), pp.107-116. https://doi.org/10.1016/j.patcog.2012.07.002.

[13] Chaudhari S \& Chatterjee S, "Detection of blood vessels in retinal images using two-dimensional matched filter", IEEE Transactions on Medical Imaging, Vol.8, No.3, (1989), pp.263-269. https://doi.org/10.1109/42.34715.

[14] Pal NR \& Pal SK, "Entropy: A new definition and its applications", IEEE transactions on systems, man, and cybernetics, Vol.21, No.5, (1991), pp.1260-1270. https://doi.org/10.1109/21.120079.

[15] Chanwimaluang T \& Fan G, "An efficient blood vessel detection algorithm for retinal images using local entropy thresholding", Proceedings of the International Symposium on Circuits and Systems, Vol.5, (2003). https://doi.org/10.1109/ISCAS.2003.1206162.

[16] Kande GB, Savithri TS \& Subbaiah PV, "Automatic detection of microaneurysms and hemorrhages in digital fundus images", Journal of digital imaging, Vol.23, No.4, (2010), pp.430-437. https://doi.org/10.1007/s10278-009-9246-0.

[17] Wu S \& Wang S, "Information-theoretic outlier detection for largescale categorical data", IEEE transactions on knowledge and data engineering, Vol.25, No.3, (2013), pp.589-602. https://doi.org/10.1109/TKDE.2011.261.

[18] Mane VM \& Jadhav DV, "Holoentropy enabled Decision tree for detection of Diabetic retinopathy", Biomedizinische Technik/ Biomedical Engineering, (2016).

[19] DIARETDB1,

http://www.it.lut.fi/project/imageret/diaretdb1/index.html. 